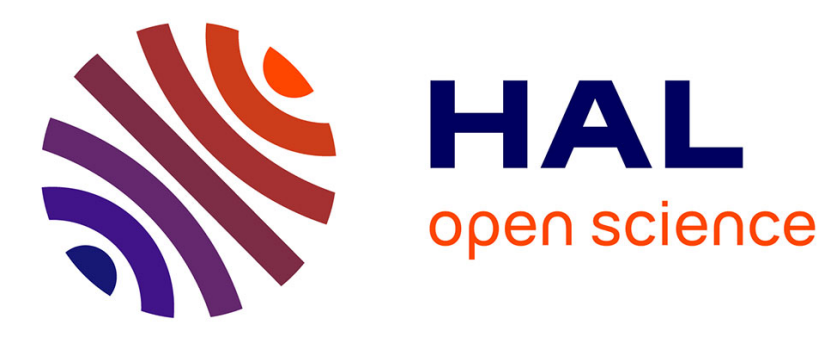

\title{
Implementation and Operation of Collaborative Manufacturing Networks
}

Jens Ziegler, Robert Buchmann, Markus Graube, Jan Hladik, Tobias Münch, Patricia Ortiz, Johannes Pfeffer, Florian Schneider, Mikel Uriarte, Dimitris Karagiannis, et al.

\section{To cite this version:}

Jens Ziegler, Robert Buchmann, Markus Graube, Jan Hladik, Tobias Münch, et al.. Implementation and Operation of Collaborative Manufacturing Networks. 15th Working Conference on Virtual Enterprises (PROVE), Oct 2014, Amsterdam, Netherlands. pp.197-208, 10.1007/978-3-662-44745-1_19. hal-01392116

\section{HAL Id: hal-01392116 \\ https://hal.inria.fr/hal-01392116}

Submitted on 4 Nov 2016

HAL is a multi-disciplinary open access archive for the deposit and dissemination of scientific research documents, whether they are published or not. The documents may come from teaching and research institutions in France or abroad, or from public or private research centers.
L'archive ouverte pluridisciplinaire $\mathbf{H A L}$, est destinée au dépôt et à la diffusion de documents scientifiques de niveau recherche, publiés ou non, émanant des établissements d'enseignement et de recherche français ou étrangers, des laboratoires publics ou privés. 


\title{
Implementation and Operation of Collaborative Manufacturing Networks
}

\author{
Jens Ziegler ${ }^{1}$, Robert Buchmann², Markus Graube ${ }^{1}$, Jan Hladik ${ }^{3}$, Tobias Münch ${ }^{3}$, \\ Patricia Ortiz ${ }^{4}$, Johannes Pfeffer ${ }^{1}$, Florian Schneider ${ }^{1}$, Mikel Uriarte ${ }^{5}$, Dimitris \\ Karagiannis $^{2}$ and Leon Urbas ${ }^{1}$ \\ ${ }^{1}$ Technische Universität Dresden, Chair of Process Control Systems Engineering, \\ 01062 Dresden, Germany \\ \{jens.ziegler, markus.graube, johannes.pfeffer, florian.schneider2, leon.urbas\} \\ @ tu-dresden.de \\ ${ }^{2}$ University of Vienna, Faculty of Computer Science, Knowledge Engineering Dpt., \\ 1090 Vienna, Austria \\ \{rbuchmann, dk\}@dke.univie.ac.at \\ ${ }^{3}$ SAP AG, Germany \\ \{jan.hladik, tobias.muench\}@sap.com \\ ${ }^{4}$ Innovalia Association, Bilbao, Spain \\ portiz@innovalia.org \\ ${ }^{5}$ Nextel Communications, Inc., Zamudio, Spain \\ muriarte@nextel.es
}

\begin{abstract}
Collaborative networks of manufacturers, suppliers and even customers are an emerging trend in global manufacturing. Higher flexibility, shorter time to market and economic as well as technological synergies create value and strengthen the market position of such virtual enterprises. The ComVantage framework already provides a sophisticated technical approach for dynamic collaborative manufacturing networks based on semantic data, mobile app orchestration, business process modelling and sophisticated access control. This article discusses the services and processes that are necessary to implement and operate a virtual enterprise using the ComVantage framework. It identifies services and service providers, and proposes an infrastructure and a tool environment for the ComVantage approach.
\end{abstract}

Keywords: Virtual enterprise, collaborative manufacturing network, mobile interaction, semantic data, co-operation, co-creation 


\section{Introduction}

In order to master the competition in a global market, companies do not only have to operate more efficiently, they further need to be much better cross-linked among each other. Moreover, agility in terms of networking and process execution is important [1]. The execution of business processes across organizational boundaries as well as crosslinking data sets of collaboration partners are key success factors and initiate the transformation of isolated individual companies towards an integrated, agile virtual enterprise. Resulting Collaborative Manufacturing Networks (CMN) may also include customers in order to leverage co-creation and co-production of customer-oriented products and services.

By definition of Barnett, et al. [2], a virtual enterprise is based on a temporary alliance of several businesses. It takes advantage of a market opportunity and dissolves, when it has passed. A virtual enterprise does not have own major resources but consists of the resources and core competencies of the individual partners. The European research project ComVantage, which is funded by the European Commission within the Framework Programme No. 7, has the goal to develop a software architecture as well as a working prototype of a distributed infrastructure for virtual enterprises [3].

From a business perspective, the most important question to be answered is which positive impacts on the business can be created with the implementation of a CMN based on the ComVantage infrastructure, and which additional expenses and dependencies must be taken into account on the opposite. Synergies during implementation may result from jointly used business services and the reuse of shared resources. Additional expenses may be incurred by the necessary provision and harmonization of data and services for the network as well as by coordinative work within the network. Most collaborative activities further create dependencies that must also be considered. In this paper, a process for the implementation and operation of a $\mathrm{CMN}$ is presented, which maximizes synergies, minimizes dependencies and clearly allocates and structures the necessary activities. The process thus minimizes business risk and ensures a structured design and operation of the network.

\section{State of the Art}

Having clarified a generalized definition of a virtual enterprise and its purposes, the interpretations and implementations of collaborative working environments are very heterogeneous Alone among publicly funded research projects, there are several projects in the field of co-operation and co-creation.

The project ADVENTURE for example has the goal to create a framework that provides tools to combine factories in a pluggable way to manufacture a particular product which consists of a high number of components from different suppliers. Core element of the three layer architecture is the central 'Dashboard portal' as the user interface for all for monitoring and managing purposes [4]. GloNet as another example follows a service-oriented approach with a novel framework for automated software service composition in a complex multi-stakeholder service scenario for product life cycles. Therefore, they provide mechanisms to model or compose software services via 
business processes and combine them with the product. The resulting product model will become available in a 'Business Services Provision Space' via web services as product-related information and services during its life cycle [5]. In contrast to product or service related virtual enterprises, BIVEE's Virtual Innovation Factory (VIF) focusses on an innovative co-creation space with an ultimate goal to transform existing production processes and organizations into new ones as a following step to the Open Innovation paradigm by Henry Chesbrough. Based on collaborative and social network tools the idea moves through a flexible life cycle, called 'Innovation Waves', allowing to cover the whole flow up to production [6].

A core aspect of a temporary virtual enterprise is their life cycle, which can be basically subdivided into the five phases of Initiation, Partner search, Process design, Execution and Dissolution as it was done by Shamsuzzoha and Helo [4]. Another common aspect is the usage of software for collaboration and communication to establish Collaborative Manufacturing Networks (CMN). They have an integrated endto-end Information and Communication Technology (ICT), formalized (business) models and a shared knowledge space, based on Web 2.0 technologies. Therefore, the network provides their partners a cloud based platform for overall operational processes, like security access control and communication, as well as certain software tools to align their own business processes, data and background systems within the shared processes and data in the CMN.

\section{The ComVantage Framework}

The ComVantage project aims at developing a software architecture and a technological foundation of generic enablers for operating a virtual enterprise. The project will provide means to evaluate the business impact and will prove the suitability of the approach in three different use cases. With respect to the challenges addressed in the introduction, the following key concepts characterize the ComVantage software architecture:

First, a decentralized approach is required in order to address the specific requirements of virtual enterprises. The ComVantage approach supports individual and local instances of a collaboration hub for each network partner. Figure 1 illustrates an exemplary CMN setup containing two partners. Each collaboration hub offers a Domain Access Server as single point of access to local datasets and a couple of local data sources. The decentralized collaboration hub concept enables each partner to keep full control about their valuable business data. Furthermore, the single point of access paradigm for each collaboration hub ensures that applications can be developed independently of specific data sources and are decoupled from their heterogeneous data models. SPARQL and JSON/RDF are used as standardized interface technology between all components of the architecture to achieve high interoperability and flexibility. However, collaboration partners are not forced to run and maintain their own infrastructure, but can use shared collaboration.

Second, semantic data harmonization based on RDF and Linked Data principles is used to realize the single point of access paradigm of the collaboration hub. Whereas 
data models (as ontologies) will be stored directly in RDF (in Triple Stores), legacy systems need to be integrated by the use of Linked Data adapters (see Figure 1). These adapters perform a mapping from legacy data (e.g. from a relational database model) to a uniform data model based on RDF for each collaboration hub. While the mappings will be defined at design-time, the extraction of data may occur at run-time in order to avoid the persistence of redundant semantic data that need to be kept in sync with the original legacy data. Additionally, a multi-tiered access control approach was developed. Cross-domain authentication, physical separation of data in views and SPARQL query rewriting based on role-driven policies ensure that unauthorized access in a setup of collaboration and inter-organizational access to information is prohibited.

Third, collaboration scenarios are driven by the execution of inter-organizational business processes to realize a cross-domain product value chain. Especially in the manufacturing industry, business processes may be very complex. On the other side, the personnel on the shop floor needs intuitive and usable mobile IT support. ComVantage has developed the Industrial App Framework that allows the orchestration of limited-purpose mobile apps to App Ensembles which support complex and interorganizational workflows. A pool of Generic Apps can be used to accelerate the user interface development. These Generic Apps can be adapted to specific use cases and automatically orchestrated to App Ensembles using a business process model.

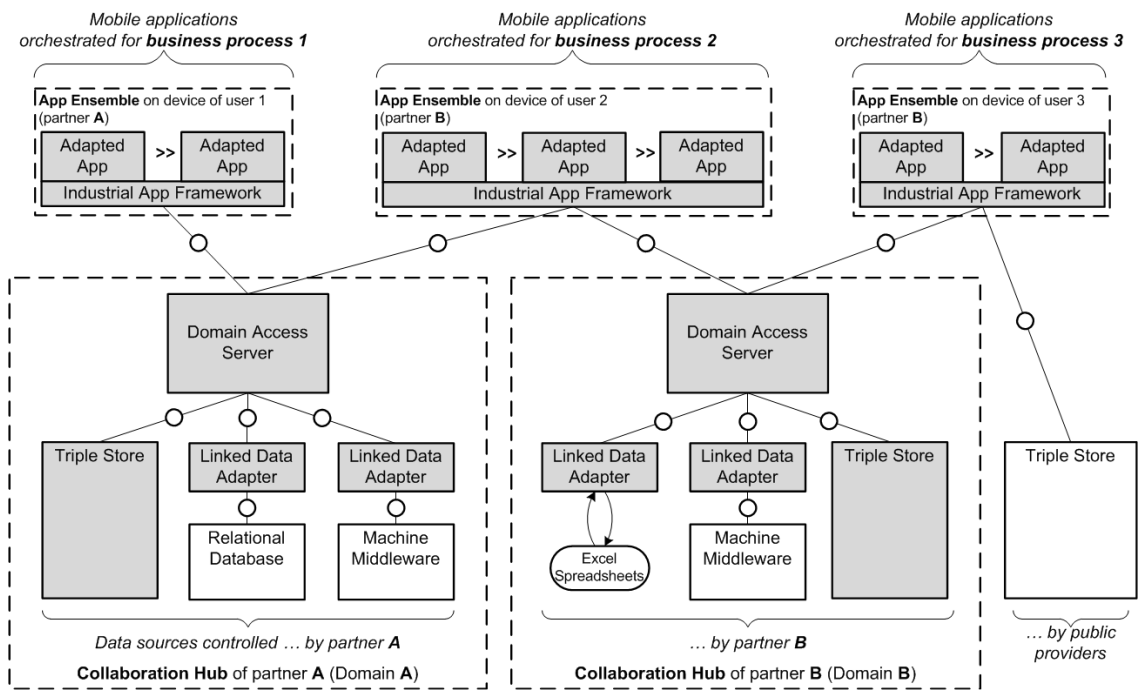

Figure 1. The big picture - Exemplary setup of a CMN with two partners and ICT infrastructure including mobile apps, collaboration hubs and data sources. 


\section{Implementation and Operation of a ComVantage Network}

\subsection{Overall Process}

In general, implementation sub-processes are categorized into collaborative processes where all partners have to jointly create a common result, shared processes that are done by an internal or external provider for all partners, and company internal processes that have to be done by each partner individually. Collaborative processes require coordinated work and joint decisions by all partners. Shared processes are equal for all partners and thus have only to be done once. The results can be shared resources or services. Company internal processes do not require collaboration. The results feed into the CMN. It is obvious that company internal processes can also be outsourced. However, the main difference to shared processes is that there are no synergy effects besides the joint use of (external) competencies.

The ComVantage overall implementation process consists of seven sub-processes, which are done sequentially in three phases. In the first phase, the common business processes (BPM) and roles (RM) are modeled. These are collaborative sub-processes, where joint decisions must be made. In the second phase, ontologies and apps are developed that are necessary to implement the BPM and RM. These are shared processes whose results are shared by all partners. In the third phase, each partner provides datasets for the developed ontologies and creates an Access Control Model (ACM) based on the RM to secure the provided data. In parallel, app ensembles are orchestrated based on the BPM, ontologies and apps. The app ensembles provide the datasets to authorized users according to the underlying BPM and are thus the final result of the entire process. All sub-processes are scalable. Individual activities may be omitted and the tools may be selected according to the actual requirements.

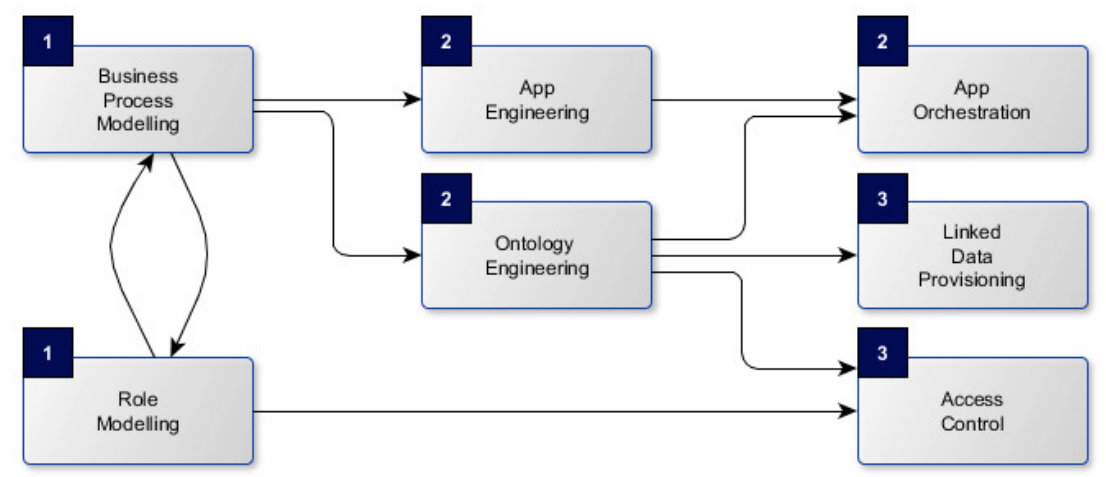

collaborative process

shared service

3 company internal process

Figure 2. Seven Steps towards a CMN - The ComVantage implementation process. 


\subsection{Role Modelling and Business Process Modelling}

The Business Process Modelling phase is traditionally tightly coupled with Role Modelling. For the hereby presented approach it is essential that role assignments use machine-readable representations so that the semantic relation between a process task and its performing role is preserved beyond the modelling phase, for example through a model serialization. To achieve this, the ComVantage approach relies on a metamodelling platform [7] and an RDF serialization vocabulary discussed in more detail in [8], with a proof-of-concept collaborative modelling tool hosted by the Open Model Initiative [9]. The modelling tool implements the iteratively evolving ComVantage modelling method, whose meta-model specifies the semantic links that can be created by modelers (usually as inter-model hyperlinks) and can be externalized for RDF queries in order to enable the later phases of the ComVantage implementation process (e.g. App Orchestration).

Business process modelling is a well-established approach, however there is a diversity of tools and languages to support it - some of them coming from standards, others from commercial tools. Popular approaches aim at high reusability across domains, therefore high abstraction levels that do not retain any domain specificity and are not prepared to collect requirements. The ComVantage approach provides the possibility of assigning, to each process task, domain specific resources and artefacts: mobile apps requirements, robots, product parts etc. Therefore, the meta-model underlying the business process modelling phase integrates with explicit associations the following facets: a) Process motivators (the trigger of a virtual enterprise - a customized product order, a service, a temporary market opportunity); b) Processes (the actual control flows triggered by the process motivators and possible acting upon them - the case of production processes); c) Process participants (liable entities or assets). In this last class, there are business or individual roles (liable entities described as a business network or organizational chart), required mobile apps (with functional
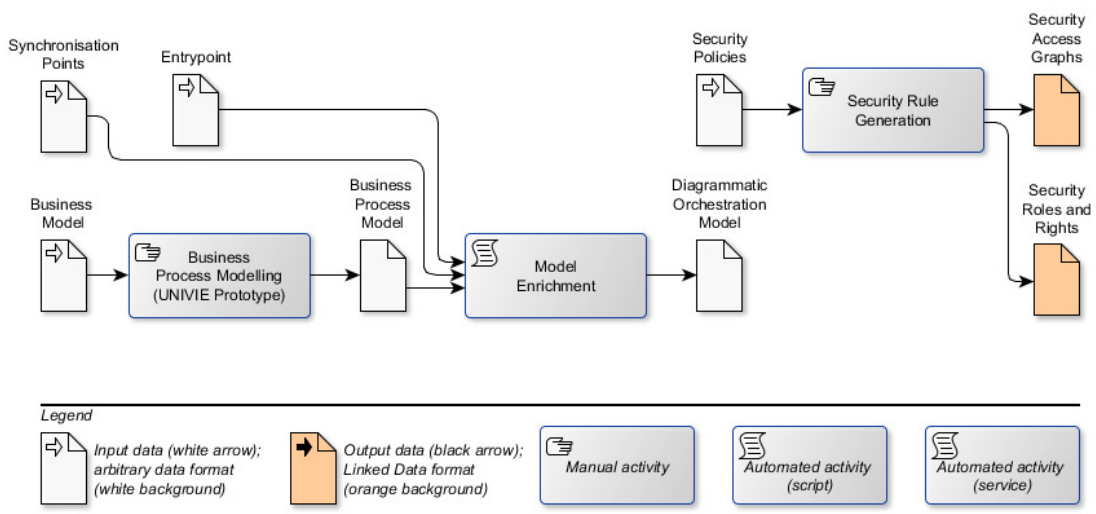

Figure 3. Role Modelling and Business Process Modelling. Activities may be manual or automated. Data may be formatted as Linked data (orange) or not (white). Input and output of the entire process marked with arrows. 
capabilities) and access means (queries indicating the kind of access and endpoint required to perform a process task). Each task of a business process model can be explicitly linked to all these types of participants, hence enabling the collection of requirements around each specific process (by querying the process model serialization). These requirements can feed into the App Orchestration and Access Control phases in the following ways. First, App Orchestration deployments can read the Diagrammatic Orchestration Model - a usage precedence graph describing which apps are required for a selected role, and in which order. This model is derived automatically from the business process model by graph transformation means (details in [8]). Second, the Access Control phase can collect the queries assigned to process tasks - they are queries that should be enabled by the policies of endpoint owners, for the specific roles linked to the same tasks.

\subsection{Ontology Engineering}

In order to design the ontologies that serve as terminologies and data structures for the applications, several ontology engineering methodologies $[10,11,12,13]$ were considered, and the Enterprise methodology developed by Uschold and King [14] was selected, since it provides sufficient guidance to provide the ontology engineers with a clear process without requiring disproportional overhead. This methodology suggests the following phases: 1 . Identifying the purpose; 2. Building the ontology, with steps a) Capturing, b) Coding, and c) Integrating existing ontologies; 3. Evaluation; 4. Documentation.

The first step can essentially be completed before the start of the CMN and laid out in the business plan. For step 2a, interviews with domain experts from the application partners have to be conducted to determine the essential terms of the corresponding domain and their relations. Step $2 \mathrm{~b}$ can be performed with the help of the Protégé tool, generating RDFS or OWL ontologies based upon these terms. In order to integrate existing ontologies (step 2c), terms from popular ontologies are used where this is possible, e.g. the FOAF and vCard ontologies describing persons and their addresses or the GoodRelations ontology for prices and other costs. Links to DBpedia entities should also be included if appropriate. Step 3 involves using the developed ontologies within the respective application areas and evaluating them with respect to usability, clarity, and consistency. The final step is performed by documenting the developed ontologies in text documents and by using the rdfs:label and rdfs:comment properties within the ontologies themselves. In order to also enable non-experts to perform basic ontology operations, the OntoSketch tool [15] was developed, which allows for refining existing ontologies by domain experts using a graphical editor.

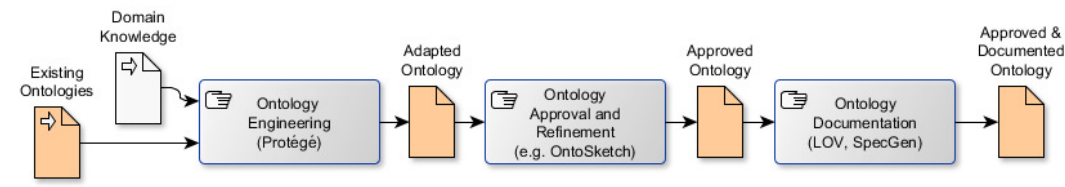

Figure 4. Describe the domain - Ontology Engineering. 


\subsection{App Engineering}

During the App Engineering phase (illustrated in Figure 5) the set of apps required for the network's business processes is created. If an app repository already exists it is first checked for apps that can potentially be reused in the business processes. Then, existing conventional apps, e.g. document viewers or messaging applications, are identified in order to integrate them during the subsequent App Orchestration phase. For all other app requests in the business processes, such as specialized data views or tasks that manipulate the Linked Data graph, new apps may be developed.

Any app that shall be used must be formally described in the developed App Description Language (ADL). The ADL is a domain specific ontology for modeling an

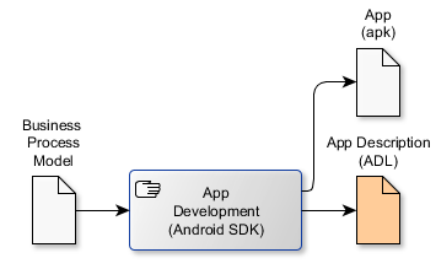

Figure 5. App Engineering. app's characteristics essential for collaboration. Besides conventional meta-data such as operating systems, supported screen sizes, languages, etc., the ADL supports modeling the app's abilities, input and output data, and entry points. The entry points are essential for forming an app network, the modeled inputs and outputs allow for interapp-communication. The abilities are later used to match apps to activities in a business process model.

\subsection{Linked Data Provisioning}

The main task of the Linked Data Provisioning phase (illustrated in Figure 6) is to generate, distribute and supply the CMN with the information necessary for the common business models. Sources of information can be, among others, Excel sheets, XML files, and proprietary engineering or control systems. The information has to be modeled with the approved ontologies in order to be useful for the CMN.

The Ontology Engineering phase delivers the meta-models of the information which is necessary for the shared business cases. Afterwards, the sources of this information (existing legacy systems or already Linked Data stores) can be identified. Linked Data adapters may be provided for this data if this information is not already present as Linked Data. The adapters use the approved ontologies from the Ontology Engineering phase to transfer the legacy data into Linked Data taking domain specific knowledge into account. If the update rate of the legacy data is rather low, simple automated model transformations can be applied which provide a RDF output for an information entity as input (e.g. a single Excel file). Various frameworks (e.g. Jena for RDF support) and application interfaces (e.g. Enterprise Server for Comos) may support this task. The RDF file is then stored in a triple store (e.g. Virtuoso) which offers access via SPARQL. If the update rate of the data is rather high (e.g. the provision of process values), a service may provide direct access to the necessary information as Linked Data. Some dynamic adapters provide a local SPARQL endpoint (e.g. XLWrap, D2RQ) whereas others only provide data via a REST web interface as Linked Data.

Usually, the majority of the data provided by the partners is only loosely coupled. Hence, a link discovery and generation process between different datasets may generate 
additional links, which will also be stored in the Linked Enterprise Data cloud. A final validation of the RDF datasets is mandatory to ensure the reliability of the information provided in the cloud. This step can be done with tools, which may perform a RDF validity check, check the instances against the used ontology or common best practices, or even check against specific defined rules. Finally, collaboration networks need information about available datasets, their structure and content. Therefore, documentation is added to all datasets, which itself can be modeled as Linked Data by using for example the VoiD vocabulary.

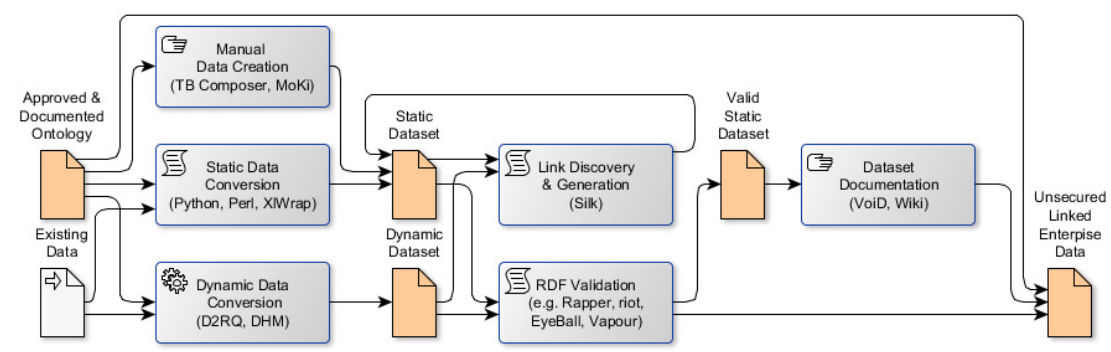

Figure 6. Breathe life into the CMN - Linked Data Provisioning.

\subsection{Access Control}

The main aim of the Access Control phase is to create an Access Control Model (ACM) based on the roles modeled (RM) to secure the information published as Linked Data. For the provisioning of trust, the ComVantage approach complements traditional XACML role-based multi-domain access control models, including SAML authentication, which are useful to control the access to dynamically changing Linked Data information, with innovative SPARQL query rewriting capabilities based on data views to address the security needs of mobile inter-organizational information sharing. The goal of the SPARQL rewriting process is to create queries that return the same results as the original queries except for those answers that contain restricted information. For this purpose, the security framework includes conditions to the original SPARQL query so that all the information requested is included within the set of information that the user is authorized to access taking into account its role, which has previously been modeled as explained in Section 4.2. In order to achieve this objective, this SPARQL rewriting process relies on the organization of the original RDF data in a set of Views with different access types (canSee or canUse) for the different user roles (see Figure 7).

The ComVantage security approach does not just guarantee that the access to the information remains secure, but it also has to ensure that the information published as Linked Data is just modified and updated by the users that are authorized to these tasks. For that purpose, SPARUL templates previously approved by the data owner are assigned to different user roles who will be allowed to modify and update the original Linked Data and the defined data views accordingly. 


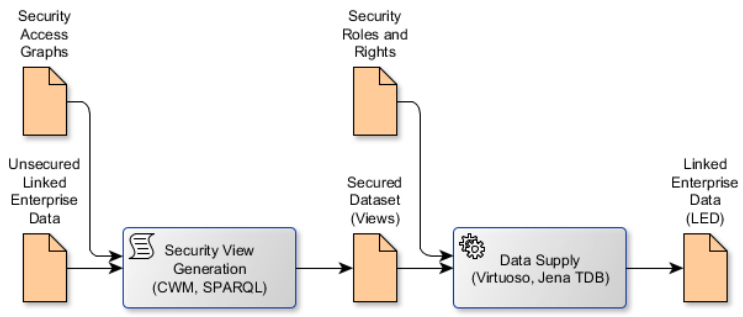

Figure 7. Secure the Linked Enterprise Data - Establishing Access Control.

\subsection{App Orchestration}

The App Orchestration phase shown in Figure 8 takes results from the Business Process Modeling phase, the Ontology Engineering phase and App Engineering phase and leverages them to create App Ensembles that are used to view and operate essential aspects of the CMN (details in [16]). They constitute the network's front end and provide means to view and manipulate the information contained in its Linked Data graph. App Ensembles are sets of Apps that are interconnected in a meaningful way to fulfill mobile support requests in business processes. They facilitate the correct switching between apps and automate the exchange of information between them. To create an App Ensemble, appropriate apps for the business process must be selected from a repository. This is accomplished by comparing requirements stated in the Diagrammatic Orchestration model (which is derived from a business process) to the app's abilities formalized in their respective App Descriptions (using the ADL). Then the apps are adapted to the data structure of the CMN. This is accomplished by assigning SPARQL templates to the apps which adhere to the networks ontologies. SPARQL templates are SPARQL queries with placeholders that fit the network's information structure and provide necessary data to the apps. Using SPARUL the templates are also employed to manipulate the Linked Data graph. In the third step, connections between the apps are established by analyzing their entry points defined in the App Descriptions. The information from the three orchestration steps is joined into a Semantic Orchestration Model and serialized as RDF.

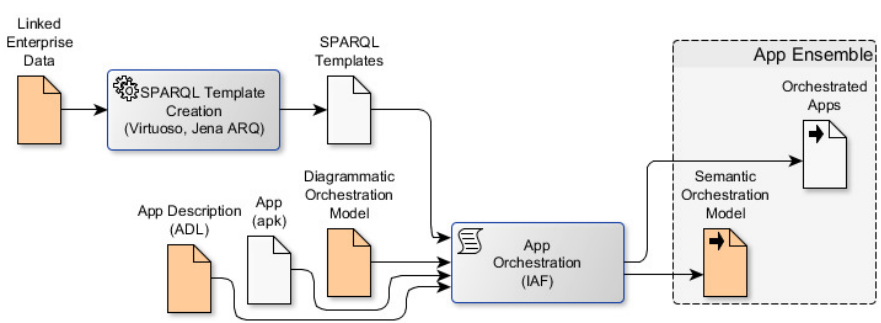

Figure 8. Bring it to the users - App Orchestration.

Installable binaries of all Orchestrated Apps together with the generated Semantic Orchestration Model form an App Ensemble. It is deployed to a mobile device where 
it can be loaded and executed using the ComVantage App Management Component that interprets the Semantic Orchestration Model.

\section{Progress beyond the State of the Art}

Both technologically and administratively, the ComVantage approach addresses the challenge to generate a verified added value through the CMN early and with scalable effort. This takes into account that CMN may differ significantly in terms of size and composition, and in terms of its business objectives. The process has therefore been designed to generate as few dependencies between the partners of the $\mathrm{CMN}$ and resolve necessary dependencies as early as possible. Thus, the implementation costs and risks are minimized. On the other hand, the process achieves maximum synergy in joint activities. It is clearly defined which results can be reused and what activities can be outsourced to service providers. Moreover, the process is scalable. Activities can be omitted if not required, tools can be selected depending on the actual requirements of the CMN. Finally, the presented process is iterative. Since the implementation of a new $\mathrm{CMN}$ and the modification of existing CMN are based on the same process, the CMN can be continuously adapted to the current requirements.

The presented process has been implemented and validated in the context of three use cases in the areas of commissioning of production lines, customer-oriented production and maintenance of process plants. Both the composition and size of the CMN (from micro-company to large enterprises, including customers) as well as its business objective (acceleration of engineering processes, maximization of customerorientation, and optimization of operation and maintenance) have been varied across the use cases. It has been found that the presented process scales well with respect to both factors. At the same time it became apparent that the flexible use of manifold tools was arduous. Also, some activities were not yet sufficiently supported by appropriate tools. A (modular) integration of the proposed tools in an integrated development environment (IDE) and the development of appropriate tools should therefore be a focus for future activities.

\section{Conclusion}

In this paper, the processes, tools and outcomes have been presented that are necessary to implement a ComVantage collaborative manufacturing network (CMN). It has been shown that the overall process strives for minimization of dependencies, maximization of synergies and great scalability of all sub processes. In the next phase of the project, suitable integrated engineering tools will be designed to better support the processes described in this paper. These tools will be directly built upon the technologies and concepts of the ComVantage framework and thus promote the feasibility of the ComVantage approach to $\mathrm{CMN}$ in industry and business. 


\section{Acknowledgments.}

The research leading to these results was partially funded by the European Community's Seventh Framework Programme under grant agreement no. FP7-284928 ComVantage.

\section{References}

1. Kim, T.Y., Lee, S., Kim, K., \& Kim, C.H.: A modeling framework for agile and interoperable virtual enterprises. In: Computers in Industry, vol. 57(3), pp. 204--217 (2006)

2. Barnett, W., Presley, A., Johnson, M., \& Liles, D. H.: An architecture for the virtual enterprise. In: Systems, Man, and Cybernetics, vol 1, pp. 506--511 (1994)

3. Muench, T., Hladik, J., Salmen, A., Altmann, W., Buchmann, R., Karagiannis, D., Ziegler, J., Pfeffer, J., Urbas, L., Lazaro, O., Ortiz, P., Lopez, O., Sanchez, E., \& Haferkorn, F.: Collaboration and interoperability within a virtual enterprise applied in a visionary mobile maintenance scenario. In: Revolutionizing Enterprise Interoperability through Scientific Foundations, pp. 137--165 (2014)

4. Shamsuzzoha, A., \& Helo, P.: Virtual enterprise architectural framework: collaboration for small and medium enterprises. In: Proc. ASME 2013 International Manufacturing Science and Engineering Conference (MSEC2013), pp. 1--8 (2013)

5. Camarinha-Matos, L. M., Macedo, P., Ferrada, F., \& Oliveira, A. I.: Collaborative Business Scenarios in a Service-Enhanced Products Ecosystem. In: Collaborative Networks in the Internet of Services, pp. 13--25 (2012)

6. Moreno, F. C., \& Escribano, C. P.: BIVEE Virtual Innovation Factory. In: CEUR Workshop Proc., vol. 1006, pp. 18--23 (2013)

7. BOC-Group, ADOxx tool, http://www.adoxx.org/live/.

8. Buchmann, R.: Conceptual modeling for mobile maintenance: the ComVantage case. In: Sprague Jr., R. H. (ed.), Proc. 47th Hawaii International Conference on Systems Sciences, IEEE CS, pp. 3390--3399 (2014)

9. The Open Model Initiative Laboratory - the ComVantage modeling prototype page, http://www.omilab.org/web/comvantage/home.

10.Fernandez-Lopez, M., Gomez-Perez, A., \& Juristo, N.: METHONTOLOGY: from Ontological Art towards Ontological Engineering. In: Proc. AAAI97 Spring Symposium Series on Ontological Engineering, pp. 33--40 (1997)

11.Grüninger, M., \& Fox, M. S.: Methodology for the design and evaluation of ontologies. In: Workshop on Basic Ontological Issues in Knowledge Sharing, Int. J. Conf. on Artificial Intelligence, pp. 1--10 (1995)

12.Lopez, M. F.: Overview of Methodologies for Building Ontologies. In: Proc. IJCAI-99 Workshop on Ontologies and Problem Solving Methods (KRR5), pp. 4.1--4.13 (1999)

13.Pinto, H. S., \& Martins, J. P.: Ontologies: How can They be Built? In: Knowledge and Information Systems, vol. 6, pp. 442--464 (2004)

14.Uschold, M.: Building Ontologies: Towards a Unified Methodology. In: Proc. Expert Systems '96, the 16th Annual Conference of the British Computer Society Specialist Group on Expert Systems, pp. 1--18 (1996)

15.Brade, M., Schneider, F., Salmen, A., \& Groh, R.: OntoSketch: Towards digital sketching as a tool for creating and extending ontologies for non-experts. In: Proc. 13th Int. Conf. on Knowledge Management and Knowledge Technologies (iKNOW'13), pp. 9--17 (2013)

16.Ziegler, J., Graube, M., Pfeffer, J., \& Urbas, L.: Beyond app-chaining: Mobile app orchestration for efficient model driven software generation. In: Proc. IEEE 17th Conference on Emerging Technologies \& Factory Automation (ETFA'12), pp. 1--8 (2012) 